\title{
Bee colony optimization with Taguchi method for solving the dynamic economic dispatch
}

\author{
Chia-Sheng Tu ${ }^{1}$, Hsi-Shan Huang ${ }^{1, *}$, Ming-Tang Tsai ${ }^{2}$, and Fu-Sheng Cheng ${ }^{2}$ \\ ${ }^{1}$ College of Intelligence Robot, Fuzhou Polytechnic, Fujian, China \\ ${ }^{2}$ Department of Electrical Engineering, Cheng-Shiu University, Kaohsiung, Taiwan
}

\begin{abstract}
Dynamic economic dispatch is to minimize the cost of power production of all the participating generators over a time horizon of 24 hours in one day. The dynamic economic dispatch with non-smooth cost functions, for which is formulated the optimal dispatch model of generations by considering the ramp up/down scheduling of power. This paper presents a Bee Colony Optimization (BCO) that applies the Taguchi Method (TM) to solve the Dynamic Economic Dispatch problem. The Taguchi method that involves the use of orthogonal arrays in estimating of the non-smooth cost function and Bee Colony Optimization is used to find the objective function under the operational of system constraints. The Taguchi method can global optimization for fast local convergence by minimizing the cost function in a few iterations. The effectiveness and efficiency of the TM-BCO is demonstrated by using a 10 unit of IEEE case with non-smooth fuel cost functions and is more effective than other previously developed algorithms. Moreover, the proposed approach presents significant computational benefits than traditional random search method especially for multi-unit systems with larger numbers of nonsmooth cost functions and more complicated dynamic economic dispatch.
\end{abstract}

\section{Introduction}

The Dynamic Economic Dispatch (DED) problem is to determine the optimal power outputs of all generating units to minimize total fuel cost while satisfying the operational constraints and load demand. DED is the complexity of search space, which the probable large number of variables in scheduling process and complicated constraints. The DED of non-smooth cost functions is a complex and high-dimensional problem with multiple constraints. A number of approaches have been developed for solving the DED problem. The two-step method is proposed to solve the DED with valve-point effect in [1] and the harmony search algorithm employed to solve the DED problem for Micro-grid in [2]. Recently, some artificial intelligent techniques have been presented to solve the DED problem and have shown their effectiveness in [3-4]. The Bee Colony Optimization (BCO) employs employed bees, onlooker bees and scout bees moving patterns to research the feasible solution in [5].

${ }^{*}$ Corresponding author:hw5400@hotmail.com 
The BCO has many of the advantages of biological intelligence in searching and it has stability in higher-dimensional search. The Taguchi Method (TM) of experimental design has been widely used in industry and the objective function will be best obtained by changes in these primary factors in the direction indicated. The [6-7] employ the Taguchi Method that involves the use of orthogonal arrays to solve the economic dispatch problem with non-smooth cost functions. The present paper applies the Bee Colony Optimization with Taguchi method to solve DED problem with non-smooth fuel cost functions. In the BCO-TM procedure, the Taguchi Method is embedded in the BCO in order to improve the behaviour patterns of each bee swarm and increase its search efficiency and accuracy in high dimensions. The TM-BCO for dynamic economic dispatch problems to increase the searching efficiency and the proposed method is feasible, robust, and more effective than previously algorithms.

\section{The problem formulation}

The dynamic economic dispatch planning of ancillary service shall satisfy the electric energy demand, and must consider whether the generators can ramp up or down the power in a short period of time. The mathematical model of power dispatch has multiple variables, which are mostly confined to the constraints of generator characteristics. The dynamic economic dispatch problem is to minimize the total operating cost. The total operating cost includes fuel cost and start cost of units which is formulated as (1).

$$
\begin{aligned}
\text { Cost } & =\sum_{t=1}^{h} \sum_{i=1}^{n} F_{i}^{t}\left(P_{i}^{t}\right) \\
& =\sum_{t=1}^{h} \sum_{i=1}^{n} a_{i} P_{i}^{t^{2}}+b_{i} P_{i}^{t}+c_{i}+\left|e_{i} \times \sin \left(f_{i}\right) \times\left(P_{i, \min }-P_{i}^{t}\right)\right|
\end{aligned}
$$

where $h$ is the scheduling hour and $n$ is the total number of generators. $P_{i}^{t}$ is the power output and $F_{i}^{t}$ is cost function of a committed unit $i$ at hour $t$. The $a_{i}, b_{i}, c_{i}, e_{i}$ and $f_{i}$ are the coefficients of unit $i$ with non-smooth cost function.

The constraints of dynamic economic dispatch problem are described as follows.

1. The load balance

$$
\operatorname{Load}^{t}=\sum_{i=1}^{n} P_{i}^{t}
$$

The sum of all $P_{i}^{t}$ should be equal to the total load demand $\operatorname{Load}^{t}$ at hour $t$.

2. The spinning reserve

$$
\text { Load }^{t}+S R^{t}=\sum_{i=1}^{n} P_{i, \max }^{t}
$$

where $S R^{t}$ is the spinning reserve and set to $10 \%$ of $\operatorname{Load}^{t}$.

3. The ramp up and ramp down constraints of generator

$$
\begin{gathered}
P_{i, u r}^{t}=P_{i}^{t-1}+U R_{i} \\
P_{i, \text { max }}^{t}=\min \left[P_{i, \max }, P_{i, u r}^{t}\right] \\
P_{i, d r}^{t}=P_{i}^{t-1}-D R_{i} \\
P_{i, \text { min }}^{t}=\max \left[P_{i, \min }, P_{i, d r}^{t}\right]
\end{gathered}
$$


$U R_{i}$ and $D R_{i}$ are the upper ramp up and lower ramp down which $P_{i, u r}^{t}$ and $P_{i, d r}^{t}$ constraints of generator of unit $i$ at hour $t-1$. The $P_{i, \max }^{t}$ and $P_{i, \min }^{t}$ are selected for $\min \left[P_{i, \max }, P_{i, u r}^{t}\right]$ and $\max \left[P_{i, \min }, P_{i, d r}^{t}\right]$.

4. Power generation limitation

$$
P_{i, \min }^{t} \leq P_{i}^{t} \leq P_{i, \max }^{t}
$$

$P_{i, \max }^{t}$ and $P_{i, \min }^{t}$ are the maximum and minimum generation limits of unit $i$ at hour $t$.

\section{The proposed methodology}

In this paper, the Taguchi Method (TM) integrated with Bee Colony Optimization (BCO) to enhance the performance of the optimization approach. This BCO mimics the food foraging behaviour of honey bees and the swarm also consists of three categories, scout bees, employed bees, and onlooker bees. The Taguchi method of experimental design is a suitable method for parameter optimization problems with a given computable and this research is to minimize a cost function.

\subsection{Bee colony optimization}

The BCO includes the employed bees, onlooker bees phases, and scout bees phases and can be described as follows.

\subsubsection{Employed bees}

The better part of the employed bees fly considering the social and cognitive information achieved by the swarm. Each bee knows its current optimal position of personal experiences and also knows the current global optimal position among all bees in the population. The employed bees try to reach compatibility between local search and global search. Each employed bee makes a change on the position of food sources to generate a new food sources in the neighbourhood and as (9).

$$
x_{e b}^{T+1}=x_{e b}^{T}+\left[c_{1} \cdot \text { rand } \cdot\left(\text { Pbest }_{e b}-x_{e b}^{T}\right)+c_{2} \cdot \text { rand } \cdot\left(\text { Gbest }-x_{e b}^{T}\right)\right] \quad e b=1,2, \cdots \cdots, N_{1}
$$

where rand is the random numbers between 0 and $1 ; c_{1}$ and $c_{2}$ are the acceleration constants in this paper set to 2.05 and 2.05; $T$ is the current iteration and $T_{\max }$ is the maximal iteration set to 200; Pbest $_{e b}$ is of the population best solution and Gbest is global optimal solution of employed bees at iteration $T$; $e b$ is the number of employed bee population and $N_{1}$ is the total number of employed bee.

\subsubsection{Onlooker bees}

The onlooker bees in the bee colony optimization will follow the employed bees to obtain nectar information and modified by using the probabilistic selection method as (10) to follow the employed bees as (11). 


$$
\begin{gathered}
\operatorname{prob}\left(x_{e b}^{T}\right)=\frac{1 / \operatorname{Cost}\left(x_{e b}^{T}\right)}{\sum_{o b=1}^{N_{1}} 1 / \operatorname{Cost}\left(x_{e b}^{T}\right)} \\
x_{o b}^{T+1}=x_{o b}^{T}+\operatorname{rand}\left(c_{1} \cdot \operatorname{rand} \cdot x_{o b}^{T}-x_{e b}^{T}\right) \quad o b=1,2, \cdots \cdots, N_{2}
\end{gathered}
$$

where $\operatorname{prob}\left(x_{o b}^{T}\right)=\left[x_{1}^{T}, x_{2}^{T}, \ldots, x_{N_{2}}^{T}\right]$ is the better Cost value at the food source; $o b$ is the number of onlooker bee population and $N_{2}$ is the total number of onlooker bee.

\subsubsection{Scout bees}

In the $\mathrm{BCO}$, the scout bees working model was modified to the average value of the global optimum solution and all solution locations as (12) and (13).

$$
\begin{gathered}
x_{s b}^{T+1}=x_{s b}^{T}+\left[\text { rand } \cdot\left(\text { Gbest }-L \cdot \text { mean }^{T}\right)\right] \quad s b=1,2, \cdots \cdots, N_{3} \\
L=\operatorname{round}(1+\text { rand })
\end{gathered}
$$

where $L$ is the average of all variable solutions in the iteration $T ; s b$ is the number of scout bee population and $N_{2}$ is the total number of scout bee. The population size for employed bees, onlooker bees, and scout bees are total population $40 \%, 40 \%$, and $20 \%$, respectively and total population size is set to 100 .

\subsection{Taguchi method}

For example, the unit in Taguchi Method of non-smooth cost function. The $F_{i}\left(P_{i}\right)$ is a cost function of several variables as Figure 1. and that is IEEE 3 units system for economic dispatch with valve point effects.
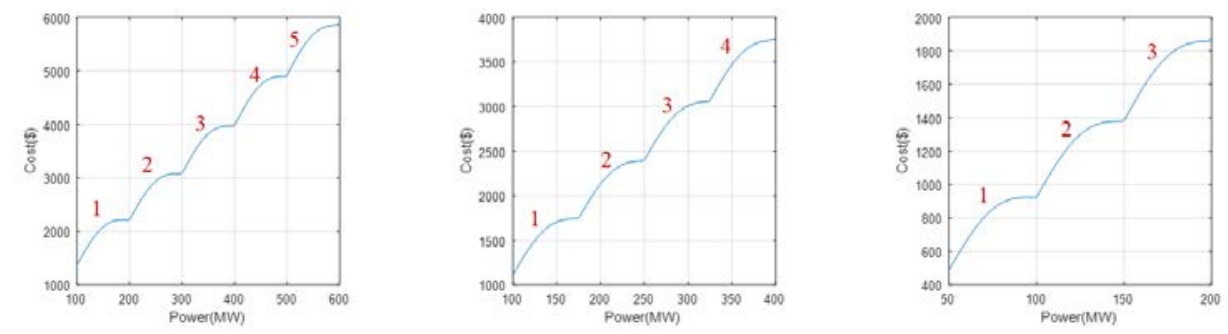

Fig. 1. Taguchi Method of non-smooth cost function.

In Taguchi method, a subset of the possible value combinations is used and to reduce the time consumed in conducting experiments. The Taguchi Method based on orthogonal arrays is introduced and chosen to provide sufficient information to determine the effects of each factor. Table 1 shows the orthogonal array shown in Taguchi Method each unit factor has different levels. In the present example, unit 1 is denoted by $P_{1,1}, P_{1,2}, \cdots \cdots, P_{1, k}=[1,2, \cdots \cdots, 5]$ for smooth cost function, the unit $i$ can be expressed as $P_{i, 1}, P_{i, 2}, \cdots \cdots, P_{i, k}=[1,2, \cdots \cdots, k]$. The Taguchi Method array in Table II is in the form of $L_{M}\left(q^{m}\right)$, where $q$ is the number of levels each factor has, $m$ is the maximum number of 
factors the table can handle, $M$ is this table total tests for the required using, the orthogonal arrays shows in Table 1.

Table 1. $L_{12}\left(5^{3}\right)$ Orthogonal arrays.

\begin{tabular}{|c|c|c|c|}
\hline Exp. & 1 & 2 & 3 \\
\hline 1 & 1 & 1 & 1 \\
\hline 2 & 1 & 2 & 2 \\
\hline 3 & 1 & 3 & 3 \\
\hline 4 & 2 & 1 & 2 \\
\hline 5 & 2 & 2 & 3 \\
\hline 6 & 3 & 1 & 3 \\
\hline 7 & 3 & 4 & 1 \\
\hline 8 & 4 & 3 & 1 \\
\hline 9 & 4 & 4 & 2 \\
\hline 10 & 5 & 2 & 1 \\
\hline 11 & 5 & 3 & 2 \\
\hline 12 & 5 & 4 & 3 \\
\hline
\end{tabular}

\subsection{Bee colony optimization with Taguchi method}

Bee colony optimization with Taguchi method for dynamic economic dispatch approach proceeds as shown in Figure 2.

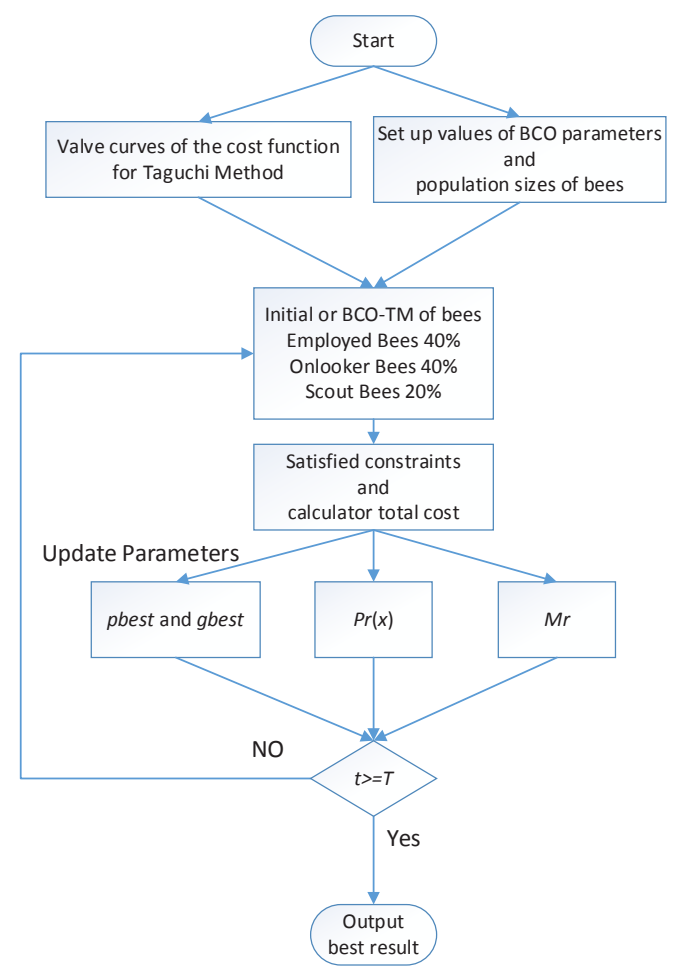

Fig. 2. The flowchart of TM-BCO. 


\section{Simulation results}

This case consists of 10 generator units considering the valve-point effects for dynamic economic dispatch. Exactly the same data of all units as given in [8] will be used in this example and the load curve of 24 hours in one day as shown in figure 3 . The algorithms were implemented in the programming language Matlab 2014b with Intel Core i7 $3.2 \mathrm{GHz}$ computer was used. Table 1 shows the results of TM-BCO programming during a total scheduling of 24 hours. The TM-BCO simulation result is given in Table 2. From Tables 2 and 3 show that the proposed algorithm did have a more robust structure and a better capability to avoid local optimum and explore new solution territories.

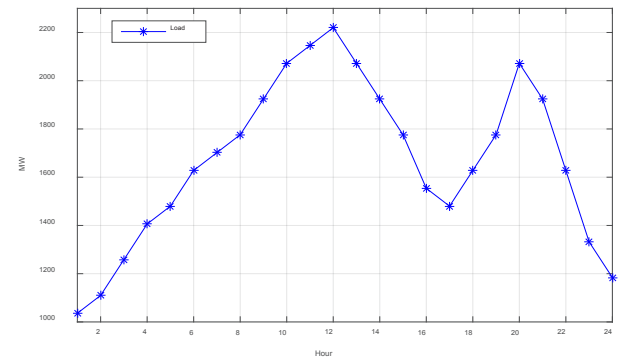

Fig. 3. The load of 24 hours in one day.

Table 2. TM-BCO simulation result for dynamic economic dispatch.

\begin{tabular}{|c|c|c|c|c|c|c|c|c|c|c|c|}
\hline \multirow{2}{*}{ hour } & \multicolumn{10}{|c|}{ Unit } & \multirow{2}{*}{ Load } \\
\hline & 1 & 2 & 3 & 4 & 5 & 6 & 7 & 8 & 9 & 10 & \\
\hline 1 & 50.00 & 1 & 73.00 & 60.00 & 73.00 & 1 & 128.19 & 47.65 & 0.00 & 5.00 & 0 \\
\hline 2 & 30.00 & 09.39 & 73.00 & 61.95 & 73.00 & 74.37 & 130.00 & 77.65 & 25.64 & 55.00 & 110.00 \\
\hline 3 & 9.31 & 3.76 & 73.00 & 60.00 & 0.88 & 7.64 & 130.00 & 7.40 & 1.01 & 5.00 & 1258.00 \\
\hline 4 & 79.31 & 298.98 & 86.06 & 60.00 & 170.88 & 160.00 & 128.37 & 47.40 & 20.00 & 55.00 & 1406.00 \\
\hline 5 & 299.31 & 323.49 & 166.06 & 60.00 & 220.88 & 132.67 & 130.00 & 72.46 & 20.14 & 55.00 & 1480.00 \\
\hline 6 & $\delta 1$ & 5 & 5 & 60.00 & 8 & 160.00 & 1 & 8 & 99 & 5.00 & 0 \\
\hline 7 & 394.44 & 305.95 & 300.15 & 68.40 & 220.71 & 160.00 & 130.00 & 47.00 & 20.35 & 55.00 & 1702.00 \\
\hline 8 & 385.05 & 385.95 & 339.64 & 68.67 & 170.71 & 160.00 & 130.00 & 60.98 & 20.00 & 55.00 & 1776.00 \\
\hline 9 & .05 & 0.00 & 03.61 & 0.77 & 217.06 & 115.51 & 00 & 47.00 & 20.00 & 55.00 & 1.00 \\
\hline 10 & 470.00 & 460.00 & 312.91 & 160.02 & 243.00 & 144.07 & 130.00 & 77.00 & 20.00 & 55.00 & 2072.00 \\
\hline 11 & 470.00 & 460.00 & 340.00 & 202.32 & 222.75 & 160.00 & 130.00 & 85.51 & 20.41 & 55.00 & 2146.00 \\
\hline 12 & 0.00 & 460.00 & 40.00 & 2.10 & 227.98 & 159.41 & 130.00 & 115.51 & 20.00 & 55.00 & .00 \\
\hline 13 & 456.52 & 460.00 & 340.00 & 192.10 & 177.98 & 153.80 & 130.00 & 86.19 & 20.41 & 55.00 & 2072.00 \\
\hline 14 & 376.52 & 395.64 & 295.09 & 142.10 & 227.98 & 160.00 & 130.00 & 116.19 & 25.48 & 55.00 & 1924.00 \\
\hline 15 & 5 & 31 & 339 & 12 & 1 & 1 & 12 & 9 & 25.46 & 55.00 & 1776.00 \\
\hline 16 & 328.79 & 235.64 & 293.48 & 118.70 & 127.98 & 160.00 & 130.00 & 84.41 & 20.00 & 55.00 & 1554.00 \\
\hline 17 & 302.06 & 309.03 & 213.76 & 68.70 & 177.98 & 148.21 & 130.00 & 54.87 & 20.40 & 55.00 & 1480.00 \\
\hline 18 & 50 & 9.03 & 93.76 & 60.00 & 127 & 73 & 130.00 & 7.00 & 20.00 & 55.00 & 1628.00 \\
\hline 19 & 457.50 & 393.76 & 269.95 & 60.00 & 177.98 & 153.79 & 130.00 & 58.02 & 20.00 & 55.00 & 1776.00 \\
\hline 20 & 470.00 & 460.00 & 340.00 & 110.00 & 227.98 & 160.00 & 130.00 & 88.02 & 31.00 & 55.00 & 2072.00 \\
\hline 21 & 464.67 & 396.27 & 340.00 & 123.42 & 177.98 & 158.63 & 130.00 & 58.02 & 20.00 & 55.00 & 1924.00 \\
\hline 22 & 384.67 & 316.27 & 314.05 & 73.42 & 127.98 & 160.00 & 129.60 & 47.00 & 20.00 & 55.00 & 1628.00 \\
\hline 23 & 304.67 & 236.27 & 240.96 & 60.62 & 46 & $15 \mathrm{~S}$ & 130 & 47.00 & 20.00 & 55.00 & 1332.00 \\
\hline 24 & 225.21 & 225.12 & 177.64 & 60.00 & 73.74 & 160.00 & 130.00 & 47.19 & 30.10 & 55.00 & 1184.00 \\
\hline
\end{tabular}


Table 3. Comparison results with methods.

\begin{tabular}{|c|c|c|c|c|c|}
\hline Methods & $\begin{array}{c}\text { Maximal } \\
\text { Converged } \\
\text { Cost (NT) }\end{array}$ & $\begin{array}{c}\text { Minimal } \\
\text { Converged } \\
\text { Cost (NT\$) }\end{array}$ & $\begin{array}{c}\text { Average } \\
\text { Converged } \\
\text { Cost (NT) }\end{array}$ & $\begin{array}{c}\text { Average } \\
\text { number of } \\
\text { generations } \\
\text { to converge }\end{array}$ & $\begin{array}{c}\text { Average } \\
\text { Execution } \\
\text { time (sec.) }\end{array}$ \\
\hline PSO & 1053155.41 & 1036159.77 & 1048154.39 & 183 & 1.11 \\
\hline BCO & 1048365.21 & 1035777.05 & 1043381.41 & 191 & 1.27 \\
\hline TM-PSO & 1042451.73 & 1032186.89 & 1036819.28 & 175 & 1.67 \\
\hline TM-BCO & 1036487.25 & 1031492.40 & 1033374.74 & 181 & 1.72 \\
\hline
\end{tabular}

Table 3 shows the maximal, minimal and average converged cost, average number of generations to converge and average execution time achieved by various methods over 100 runs. This paper uses TM-BCO to calculate the minimal converged cost is 1031492.40(NT\$) and average execution time is 1.72 (sec.). Table 4 shows the PSO, BCO, TM-PSO and TM$\mathrm{BCO}$ of 24 hours minimal cost for dynamic economic dispatch. The convergence characteristics for PSO, BCO, TM-PSO and TM-BCO as shown in Figure 4.

Table 4. Simulation methods.

\begin{tabular}{|c|c|c|c|c|}
\hline \multirow{2}{*}{ hour } & \multicolumn{4}{|c|}{ Cost (NT\$) } \\
\hline & PSO & $\mathrm{BCO}$ & TM-PSO & TM-BCO \\
\hline 1 & 29114.02 & 28407.65 & 28346.71 & 28366.00 \\
\hline 2 & 30633.91 & 30527.17 & 30626.08 & 30733.43 \\
\hline 3 & 33808.95 & 33600.48 & 33468.29 & 33650.64 \\
\hline 4 & 37110.60 & 37378.18 & 37021.51 & 36840.56 \\
\hline 5 & 38299.45 & 38553.47 & 38818.59 & 38805.25 \\
\hline 6 & 41813.25 & 41583.53 & 42197.28 & 41703.79 \\
\hline 7 & 43333.25 & 43536.87 & 43633.23 & 43225.63 \\
\hline 8 & 45074.65 & 45238.67 & 44650.71 & 45374.64 \\
\hline 9 & 48865.07 & 48719.45 & 48384.47 & 48474.59 \\
\hline 10 & 52392.50 & 52590.43 & 52455.52 & 52583.82 \\
\hline 11 & 54428.73 & 54539.42 & 54312.35 & 53970.65 \\
\hline 12 & 56369.91 & 55847.45 & 56046.31 & 55817.13 \\
\hline 13 & 52326.79 & 52379.08 & 52509.16 & 52137.67 \\
\hline 14 & 48977.08 & 49014.90 & 48628.64 & 48634.18 \\
\hline 15 & 45977.13 & 45608.79 & 45598.94 & 45569.79 \\
\hline 16 & 41200.17 & 40462.33 & 40469.53 & 40537.55 \\
\hline 17 & 38765.64 & 38728.77 & 38838.81 & 38663.78 \\
\hline 18 & 42413.83 & 41593.75 & 41980.78 & 41281.98 \\
\hline 19 & 44949.14 & 45443.06 & 45004.72 & 45029.77 \\
\hline 20 & 52378.54 & 52535.34 & 52130.21 & 52472.83 \\
\hline 21 & 48520.42 & 48807.13 & 48685.71 & 48514.70 \\
\hline 22 & 41846.32 & 42082.17 & 41536.21 & 41714.50 \\
\hline 23 & 35504.70 & 35902.82 & 34976.91 & 35437.72 \\
\hline 24 & 32055.71 & 32696.14 & 31866.21 & 31951.79 \\
\hline Total & 1036159.77 & 1035777.05 & 1032186.89 & 1031492.40 \\
\hline
\end{tabular}




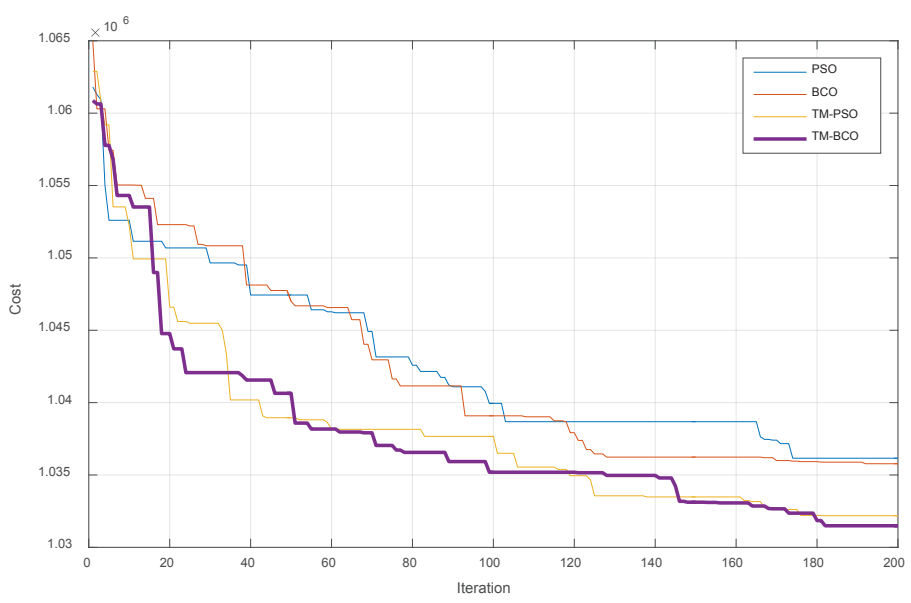

Fig. 4. The convergence characteristics for method.

\section{Conclusions}

The Bee Colony Optimization with Taguchi Method is capable of solving the constrained dynamic economic dispatch problem. The TM-BCO was incorporated the orthogonal arrays to select a better unit factor, consequently enhancing BCO. The TM-BCO can provide a powerful global exploration capability of IEEE 10 units. From test result proved the effectiveness of the proposed algorithm in solving dynamic economic dispatch problems under a reasonable execution time. The TM-BCO has great potential for further applications for solving the economic dispatch problems in real-world applications.

\section{References}

1. Pengpeng Yang, Chunping Zhu, Long Zhao, Mingqiang Wang, Xingyao Ning, Yuan Liu, 2016 IEEE Region 10 Conference, (2016)

2. Ritesh Jha, Nand K. Meena, Anil Swarnkar, Nikhil Gupta, K. R. Niazi, 2015 Annual IEEE India Conference, (2015)

3. Taher Niknam, Farank Golestane, Bahman Bahmanifirouzi, 2011 IEEE Power Engineering and Automation Conference, 1 (2011)

4. A. Bakirtzis, V. Petridis, S. Kazarlis, IEE Proceedings-Generation, Transmission and Distribution, 141 (1994)

5. D. Karaboga, B. Akay, Journal of Applied Mathematic Computation 2009, (2014)

6. Ying Cai, Derong Liu, Proceedings of the 44th IEEE Conference on Decision and Control, (2005)

7. Derong Liu, Ying Cai, IEEE Transactions on Power Systems, 4 (2005)

8. Yan Chen, Jinyu Wen, Lin Jiang, Shijie Cheng, IET Generation, Transmission \& Distribution, 7 (2013) 